\title{
Vol. 70, No. 3
}

In the report "Vaccination Coverage with Selected Vaccines and Exemption Rates Among Children in Kindergarten -

United States, 2019-20 School Year," on page 77, in the Table, for the row for Kansas, in the columns labeled "MMR 2 doses," "DTaP 5 doses," and "Varicella 2 doses," the percentages should have been $\mathbf{9 0 . 0} \%, \mathbf{8 9 . 7 \%}$, and $\mathbf{8 9 . 1 \%}$, respectively. 